\title{
Discriminación y violencia de género en estudiantes politécnicos en Chimborazo - Ecuador @(1) (0)
}

(Discrimination and gender violence in polytechnic students in Chimborazo Ecuador)

Kathy Violeta Serrano Avalos. ${ }^{1}$, Lilia del Carmen Villavicencio Narváez. ${ }^{2}$, Martha Cecilia Mejía Paredes. ${ }^{3} \&$ Dayanara de los Ángeles Peñafiel Salazar. ${ }^{4}$

\begin{abstract}
.
DOI: https://doi.org/10.33262/cienciadigital.v3i2.6.521

Gender violence is a serious social problem that affects women of different ages, social classes, cultures or academic levels and that overcomes existing stereotypes regarding those who suffer it, why and where it occurs. Thus, in recent years, this problem has increased worldwide. In Ecuador, the figures do not lie about gender violence; in 2017 more than a hundred women were already killed by their partners or former partners, crimes that fit within the figure of femicide. This figure is alarming because in 2016 there were 8 , which shows that the numbers are doubled. Faced with this problem, this investigation of gender discrimination and violence is carried out with students of Public Health School at the Escuela Superior Politecnica de Chimborazo in Ecuador, in order to identify attitudes, practices of discrimination and physical, psychological and social violence. The investigation was descriptive of cross-sectional type; a survey was elaborated for the information and validated by experts, a group of similar characteristics to the study group and by the Cronbach method. The main results indicated that in the faculty of Public Health, students have suffered physical violence by other students and psychological violence by teachers; $8 \%$ of students admit to have
\end{abstract}

\footnotetext{
${ }^{1}$ Escuela Superior Politécnica de Chimborazo, Facultad de Salud Pública, Riobamba, Ecuador. kathy.serrano@espoch.edu.ec

${ }^{2}$ Escuela Superior Politécnica de Chimborazo, Facultad de Salud Pública, Riobamba, Ecuador. lilia.villavicencio@espoch.edu.ec

${ }^{3}$ Escuela Superior Politécnica de Chimborazo, Facultad de Salud Pública. Riobamba, Ecuador. martha.mejia@espoch.edu.ec

${ }^{4}$ Escuela Superior Politécnica de Chimborazo, Facultad de Salud Pública. Riobamba, Ecuador. dayanara.penafiel@espoch.edu.ec
} 
suffered physical and psychological violence from their partners. Further dissemination of the regulations and actions that ESPOCH is developing in response to this problem is recommended so that they are better known and act with integral strategies in the prevention of gender violence.

Keywords: Gender, Gender Violence, Discrimination, Students

\section{Resumen.}

La violencia de género es un grave problema social que afecta a mujeres de diferentes edades, clases sociales, culturas o niveles académicos que superan estereotipos existentes respecto a quienes la sufren, por qué y dónde se produce. Es así que en los últimos años esta problemática ha aumentado a nivel mundial. En Ecuador las cifras no mienten sobre violencia de género; en el 2017 más de un centenar de mujeres ya fueron asesinadas por sus parejas o ex parejas, delitos que caben dentro de la figura de femicidio. Pero esta cifra alarma porque en 2016 fueron 8, lo cual muestra que los números se duplicaron. Frente a esta problemática se realiza esta investigación de discriminación y violencia de género en los estudiantes de la Facultad de Salud Pública de la Escuela Superior Politécnica de Chimborazo en Ecuador, con el objetivo de identificar actitudes y prácticas de discriminación y violencia física, psicológica y social. La investigación fue de tipo descriptiva de corte transversal, para la recolección de la información se elaboró una encuesta la misma que fue validada por expertos, un grupo de similares características al grupo de estudio y mediante el método de Cronbach. Los principales resultados indicaron que dentro de la facultad de Salud Pública los estudiantes han sufrido violencia física por parte de otros estudiantes y violencia psicológica por parte de docentes; el 8\% de estudiantes reconoce haber sufrido violencia física, psicológica por parte de sus parejas. Se recomienda mayor divulgación de la normativa y acciones que la ESPOCH está desarrollando frente a esta problemática para que sean difundidas y se actúe con estrategias integrales en prevención de la violencia de género.

Palabras claves: Clave: Género, Violencia De Género, Discriminación, Estudiantes

\section{Introducción.}

La violencia de género es una problemática social que afecta a mujeres de diferentes edades, clases sociales, culturas o niveles académicos y que supera estereotipos existentes respecto a quienes la sufren, por qué y dónde se produce. Como parte de esta realidad, los datos de diversas investigaciones internacionales indican que también es un fenómeno que se da en el ámbito universitario. 
Este problema social comenzó con el patriarcado que situaba a las mujeres por debajo de los hombres, haciéndolas ver como inferiores, débiles, sin capacidad para poder expresar su opinión en ningún ámbito ya sea social como familiar, es una realidad no solo en nuestro país sino a nivel mundial, dando un giro de noventa grados queriendo en la actualidad frenar con tantas desgracias que no solo afectan a la víctima sino a todo su entorno familiar.

Con los años las relaciones de poder históricamente desiguales se fortalecen dando origen a varias manifestaciones de violencia entre género, a la dominación y a la discriminación de la mujer por parte del hombre, según las Naciones Unidas se define al feminicidio como: " el asesinato de mujeres como resultado extremo de la violencia de género, que ocurre tanto en el ámbito privado como en el público y, comprende aquellas muertes de mujeres a manos de sus parejas, ex parejas o familiares, asesinadas por acosadores, agresores sexuales y/o violadores, así como aquellas que trataron de evitar la muerte de otra mujer y quedaron atrapadas en la acción femicida".

Para ello las Naciones Unidas, El Consejo Nacional de Mujeres, La Dirección Nacional de Genero, La Convención para la Eliminación de todas las formas de discriminación contra la mujer, CEDAW, La Conferencia Mundial de los Derechos Humanos, Declaración y Programa de Viena, La Declaración y Plataforma de Acción de Beijing y la Legislación Nacional de cada país permite adquirir compromisos para erradicar la violencia de género, resolver los problemas sociales, económicos y políticos del mundo que afecten la equidad de género.

Según la Encuesta Nacional sobre Relaciones Familiares y Violencia de Género en el Ecuador 6 de cada 10 mujeres mencionaron haber sido víctimas de una o más formas de violencia de género, ya sea física, psicológica o sexual, por parte de cualquier persona conocida o extraña, cada media hora se registran denuncias sobre violencia intrafamiliar, es menester que se aplique una ley que promueva y erradique la violencia de género en nuestro país.

Es evidente que la violencia de género es un problema social complejo que afecta a mujeres de diferentes edades clases sociales, culturas o niveles académicos y supera estereotipos respecto a quienes la sufren porque y donde se produce siendo la primera causa de mortalidad entre las mujeres antes de los cuarenta y cinco años.

Las investigaciones en el ámbito Internacional corroboran que este fenómeno también se da en el contexto universitario, uno de los retos más importantes que enfrentan las instituciones de Educación Superior es el de erradicar la desigualdad de género que todavía se manifiesta y reproduce a su interior. Desde sus orígenes, las universidades han sido espacios históricamente desfavorables para las mujeres, quienes durante siglos ni siquiera tuvieron derecho a acceder a este nivel educativo. 
Esta investigación parte de una necesidad institucional tiene como propósito contribuir a la igualdad de género en la comunidad politécnica, no solo llamar la atención sobre la necesidad de hacer cumplir medidas para prevenir y erradicar la violencia contra la mujer e informar acerca de la incidencia de este tema en nuestro país. Durante la presente investigación podremos determinar las principales formas de discriminación y violencia de género en el sector estudiantil de la ESPOCH.

\section{Metodologia.}

El estudio es de tipo descriptivo (cuantitativo y cualitativo). La población de estudio está constituida por los, estudiantes de la facultad de Salud Pública. La información requerida se obtendrá de fuentes primarias mediante la aplicación de una encuesta dirigida a la muestra seleccionada.

La realización de la investigación tuvo dos fases:

\section{Primera Fase}

a) Realización de un estudio bibliográfico sobre la temática de la violencia de género. Utilizando el Google Académico se pudieron determinar un total de 70 referencias que contenían el término "violencia de género" en el título del documento y publicados durante los años 2000 al 2017. Ello permitió precisar artículos de importancia en relación con el tema objeto de estudio.

b) Utilización de los datos reportados en Ecuador en relación con la violencia de género. La "Encuesta Nacional sobre Relaciones Familiares y Violencia de Género contra las Mujeres" fue la Base de Datos utilizada (INEC, 2012). La misma tuvo por objetivo generar datos y producir información estadística sobre la prevalencia, incidencia y magnitud de los distintos tipos de violencia de género (física, psicológica, sexual, patrimonial) que sufren o han sufrido las mujeres, tanto en el ámbito público (escolar, laboral y social) como en el privado (hogar, familia, relación de pareja); sobre el conocimiento o búsqueda de servicios e instancias de justicia para enfrentar la violencia; y sus percepciones sobre la respuesta institucional. (INEC, 2012).

\section{Segunda Fase:}

c) La revisión de la misma permitió elaborar un instrumento de recolección de información necesaria para la investigación, el que cuenta con 28 preguntas, el mismo que fue validado mediante el método de Cronbach.

d) El procedimiento para la recolección de la información fue la aplicación de la encuesta a la muestra seleccionada, estudiantes de la facultad de Salud Pública de la ESPOCH.

e) La información recopilada, se procesó utilizando tablas y gráficos a través del programa estadístico SPSS. 
La técnica de producción de datos utilizada es el cuestionario estructurado cerrado y el universo de estudio, de la Facultad de Salud Pública (estudiantes) es de 2550 personas. El diseño muestral para un nivel de confianza del $95 \%$ y un error de $5 \%$, con $p=40$ y q $=60$, requería un tamaño mínimo de 160 estudiantes.

Se pretendió explorar la interpretación que hacen los estudiantes sobre violencia y discriminación de género y, por otro, ahondar en el sentido que otorgan a sus experiencias y vivencias en el mundo de los sentimientos y las relaciones de pareja. Esto es, en esta parte de la investigación, no se ha buscado obtener una descripción y un análisis estadísticamente representativo de las opiniones de los y las jóvenes sobre la violencia de género y el mundo de los afectos o sus concepciones de la pareja.

\section{Confiabilidad del Instrumento de Investigación.}

Previa a la construcción final del instrumento, se aplicó el procedimiento del cálculo e interpretación del Alfa de Cronbach para el caso de validación de la consistencia interna del cuestionario, es un procedimiento que permite evaluar si se recopila información defectuosa o si el instrumento realiza mediciones fiables; es decir estables y consistentes, al igual que la homogeneidad de las preguntas, en base a la interpretación el indicador del valor de 1 éste indica la mejor fiabilidad y los diferentes autores manifiestan que a partir de 0,7 se considera un valor aceptable de confiabilidad del instrumento.

Se redactaron 28 preguntas con el objetivo de identificar aspectos sobre la violencia de género en los estudiantes politécnicos, con la finalidad de realizar acciones encaminadas a mitigar los posibles casos de violencia. Las preguntas $1-5,8,11,16-28$ presentaron cuatro opciones, nombradas con los literales: a, b, c, d. Las preguntas 6, 7, 12 ofrecieron cinco opciones $(\mathrm{a}, \mathrm{b}, \mathrm{c}, \mathrm{d}, \mathrm{e})$. La forma de diseño se orientó para que se escoja una sola opción, la que se considere correcta.

La fórmula empleada fue mediante la varianza de los ítems o preguntas. (Cronbach, 1951). El manejo de los datos fue facilitado por el cálculo que se realizó con Microsoft Excel. Para el cálculo de la fórmula se volcaron los resultados a una tabla de datos, para lo que se trasladaron las respuestas obtenidas por parte de la aplicación a 30 estudiantes, Según varios autores se recomienda que se trabaje con mínimo de 30 individuos para aplicar el proceso de validación de Cronbach; es decir se acató esta recomendación.

Se procedió al cálculo de la varianza de cada pregunta y a la sumatoria de las varianzas para su reemplazo en la fórmula.

El índice resultado de la aplicación de la fórmula es de 0.73 . La escala establece valores del 0 a 1 de confiabilidad, que se refiere a la estabilidad o consistencia de los resultados obtenidos; es decir: "el grado en que la aplicación repetida del instrumento, al mismo sujeto, produce iguales resultados". 
En base a lo referido por Oviedo y Campo (2005:577), que manifiestan: “El valor mínimo aceptable para el coeficiente alfa de Cronbach es 0,70; por debajo de ese valor la consistencia interna de la escala utilizada es baja" Es decir, según este criterio el coeficiente se considera tolerable si supera el 0,70.

En Conclusión para el intrumento de Violencia de Género de la presente investigación, el coeficiente resultante es de $\underline{0.73}$; por lo que se concluye que se considera aceptable, es decir el cuestionario es fiable, hace mediciones estables y consistentes y las preguntas utilizadas son homogéneas

\section{Resultados.}

Los principales resultados indicaron que dentro de la facultad de Salud Pública los estudiantes han sufrido violencia física por parte de otros estudiantes y violencia psicológica por parte de docentes; el $8 \%$ de estudiantes reconoce haber sufrido violencia física, psicológica por parte de sus parejas.

Tabla1. Ámbitos en los que se presentan mayor número de casos de maltrato o violencia de género

\begin{tabular}{ccc}
\hline \multirow{2}{*}{ OPCIÓN } & \multicolumn{2}{c}{ Estudiantes } \\
\cline { 2 - 3 } & $\mathrm{N}$ & $\%$ \\
\hline Familiar & 112 & 70.00 \\
Laboral & 3 & 1.88 \\
Educacional & 33 & 20.63 \\
Social & 12 & 7.50 \\
\hline
\end{tabular}

Fuente: Encuestas a estudiantes

Elaborado por: Investigadores.

La tabla 1 muestra que existe un mayor porcentaje de violencia de género dentro del ámbito familiar (70\%). El 20,63 \% de los estudiantes afirma recibir algún tipo de maltrato en el ámbito educacional.

Tabla 2. Tipos de violencia recibida por los estudiantes

\begin{tabular}{lcc}
\hline & \multicolumn{2}{c}{ Estudiantes } \\
\cline { 2 - 3 } & $\mathrm{N}$ & $\%$ \\
\hline Física & 24 & 15.00 \\
Psicológica & 26 & 16.00 \\
Sexual & 12 & 7.50 \\
\hline
\end{tabular}

Fuente: Encuestas a estudiantes

Elaborado por: Investigadores. 
De acuerdo a la información obtenida se pudo determinar que alrededor del 39\% de estudiantes ha recibido algún tipo de violencia, el 15\% afirmó haber sufrido violencia física, el 16\% agresión psicológica, principalmente insultos, burlas o críticas (tabla 2).

Tabla 3. Violencia recibida por tipo de perpetrador

\begin{tabular}{lcc}
\hline & Física & Psicológica \\
\cline { 2 - 3 } & $\%$ & $\%$ \\
\hline Otro & 15 & 11 \\
estudiante & 8 & 8 \\
Pareja & - & 5 \\
Docente & - & \\
\hline
\end{tabular}

Fuente: Encuestas a estudiantes Elaborado por: Investigadores.

Al investigar sobre la persona que perpetra el maltrato, se observó que el mayor porcentaje de estudiantes refiere haber recibido violencia por parte de otro estudiante, tanto física (15\%), traducida en empujones, sacudidas y lanzada de objetos; así como violencia psicológica $(11 \%)$. Un menor porcentaje $(8 \%)$ menciona haber sufrido maltrato por parte de su pareja (bofetadas, golpes, heridas) y el 5\% reporta violencia psicológica, por parte de algún docente (desprecio, desvalorización y rechazo) (Tabla 3).

Tabla 4. Conocimiento sobre rutas de atención para prevención de violencia

\begin{tabular}{lcc}
\hline \multirow{2}{*}{ OPCIÓN } & \multicolumn{2}{c}{ Estudiantes } \\
\cline { 2 - 3 } & $\mathrm{N}$ & $\%$ \\
\hline $\begin{array}{l}\text { Conoce } \\
\text { Ha }\end{array}$ & 50 & 31.25 \\
$\begin{array}{l}\text { escuchado } \\
\text { pero no sabe } \\
\text { quien }\end{array}$ & 72 & 45.00 \\
$\begin{array}{l}\text { Le es } \\
\text { indiferente } \\
\text { saber }\end{array}$ & & \\
No conoce & 18 & 11.25 \\
\hline $\begin{array}{l}\text { Fuente: Encuestas a estudiantes } \\
\text { Elaborado por: Investigadores. }\end{array}$ & \\
\end{tabular}

Fue importante dentro de la investigación identificar si dentro de la institución educativa existen rutas de atención para víctimas de violencia de género, en este sentido, se determinó la existencia de un protocolo de actuación para la prevención, seguimiento y sanción de acoso sexual y otras formas de violencia basada en género de la ESPOCH. A pesar de ello, se evidenció que solamente un $31 \%$ de estudiantes conoce sobre la existencia de rutas de 
atención o acciones de prevención de violencia dentro de la institución y un $45 \%$ ha escuchado sobre aquello pero no conoce quien lo brinda o lo realiza. (Tabla 4).

Tabla 5. Lugar de preferencia para denuncias de maltrato

\begin{tabular}{lcc}
\hline \multirow{2}{*}{ OPCIÓN } & \multicolumn{2}{c}{ Estudiantes } \\
\cline { 2 - 3 } & $\mathrm{n}$ & $\%$ \\
\hline Policía & 73 & 45.63 \\
Autoridades & & \\
de la & 7 & 4.38 \\
Politécnica & & \\
Fiscalía & 37 & 23.13 \\
Ecu 911 & 38 & 23.75 \\
Otros & 5 & 3.13 \\
\hline $\begin{array}{l}\text { Fuente: Encuestas a estudiantes } \\
\text { Elaborado por: Investigadores. }\end{array}$
\end{tabular}

Cuando se presentan casos de violencia de género el $45.63 \%$ de estudiantes manifiesta que prefiere informar a la Policía, el 23\% a la Fiscalía y al Ecu 911 un 23\% (Tabla 5).

\section{Conclusiones.}

- El $15 \%$ de los estudiantes afirman haber recibido violencia física por parte de otro estudiante, principalmente; empujones, sacudidas y lanzada de objetos; es decir se advierte la presencia de bullying.

- Los estudiantes indican haber sufrido algún tipo de violencia psicológica en el último año dentro de la institución, el 11\% dice haber sufrido agresión psicológica, principalmente (insultos, burlas, críticas) de otros estudiantes y el 5\% reporta violencia psicológica por parte de docentes (desprecio, desvalorización y rechazo).

- En relación a ser víctimas de violencia por parte de la pareja en el último año el 8\% de estudiantes que tienen pareja manifiestan que han sufrido violencia física (bofetadas, golpes, heridas y moretones) y psicológica.

- Según los resultados obtenidos se puede concluir que la mayoría de los estudiantes desconocen sobre la normativa, las políticas o acciones que se desarrollan en la institución en temas de prevención de discriminación y violencia, además desconocen sobre rutas de atención para víctimas de violencia basada en género.

\section{Referencias bibliográficas.}

Alcázar Miguel Ángel, Gómez Gregorio. 2001. Aspectos psicológicos de la Violencia de Género una propuesta de intervención, Vol1,No 2, pp 33-49 
Ávila, Francisco. Universidad Nacional Experimental Rafael María Baralt. Historia de las universidades. Venezuela. [1997.]. Disponible en: http://www.geocities.com/Athens/Acropolis/6708/universidad1.htm.

Barros Carvajal, V., Chóez Chiliquinga, E., Zambrano Pérez,. F. (2019) Síndrome del hombre maltratado y la violencia intrafamiliar. Ciencia Digital, 3,1.1

Bolaños Cubero. C (2011). La calidad universitaria desde una perspectiva de género vol. 5: número especial (setiembre)doi 10.15517/aie.v5i4.9181

Celina Oviedo, H; Campo Arias, A.2005. Aproximación al uso del coeficiente alfa de Cronbach. Revista Colombiana de Psiquiatría, XXXIV pp 572-580. Disponible en: http://www.redalyc.org/articulo.oa?id=80634409

Camacho, Gloria. (2014). La violencia de género contra las mujeres en el Ecuador: Análisis de los resultados de la Encuesta Nacional sobre Relaciones Familiares y Violencia de Género contra las Mujeres. Disponible http://repositorio.dpe.gob.ec/handle/39000/2153

Expósito Francisca. 2011. Violencia de género. La asimetría social en las relaciones entre mujeres y hombres favorecen la violencia de género. Es necesario abordar la verdadera causa del problema: su naturaleza ideológica. Revista Mente y Cerebro Investigación y Ciencia, pp 20-25. Disponible en: www.investigacionyciencia.es/revistas/mente-y...sociales.../violencia-de-gnero8894

Logroño, Julieta. (2009). Situación de acoso, abuso y otros delitos sexuales en el ámbito de la educación superior. Caso Universidad Central de Ecuador. Quito: Consejo Nacional de $\quad$ Mujeres. 157 p. $\quad$ Disponible: http://repositorio.iaen.edu.ec/handle/24000/4373

Mato, Daniel. 2007. Interculturalidad y educación superior: diversidad de contextos, actores, visiones y propuestas. Revista Nómadas, no. 27. Universidad Central de Colombia.

Monzón, Ana Silvia 2008. En la academia, las mujeres cuentan. Diagnóstico y criterios para el diseño e implementación del "Sistema Universitario de Estadísticas de Género y Etnia-SUIES.

Moreyra - Navarrete M. 2005. Coeficiente alfa de Cronbach. Validación del instrumento de recolección de datos. Universidad César Vallejo. Disponible en: http://slideplayer.es/slide/3292377/

Novillo Solorzano, V., Molina Cusme , J., Vicuña Zambrano, Y., Vizuela Carpio, J., Velasteguí López, E (2017) Promover la aplicación de políticas de inclusión en el 
proceso de formación académica en instituciones de Educación Superior del Ecuador. Ciencia Digital, 1, 1 DOI: https://doi.org/10.33262/cienciadigital.v1i1.51

Priscila Romero. Notimundo Violencia de Género en el Ecuador. [Internet] 2017. [citado 28 de enero del 2017]. Disponible en: https://notimundo.com.ec/la-violencia-de-generoen-ecuador/

Pontón Cevallos, Jenny. Femicidio en el Ecuador: realidad latente e ignorada (Tema central). En: Ciudad segura, Quito: FLACSO Sede Ecuador, (n.31, 2009): pp. 4-9. Disponible: http://hdl.handle.net/10469/286

Programa Universitario De Estudios De Género-Universidad Nacional Autónoma De México (PUEG-UNAM) (2011). Encuesta sobre la situación de mujeres y hombres en la UNAM (CU), 2009-2010. México: Universidad Nacional Autónoma de México.

Test para frenar, detectar y prevenir la violencia de género. Disponible en: https://web.ua.es/es/dia-violencia-genero/documentos/25n.../campana-semaforo.pdf

Vega Esmeralda de la Moscoso, Verónica Santillán, Janet, Ideología Religiosa, Mujer y Violencia en la Sierra Ecuatoriana", Impreso s/n, Quito.

Yungan Yungan, R., Reinoso Naranjo, E., Chifla Guato, L., Araujo Vasconez, S. (2019) La violencia psicológica en el ámbito educativo. Ciencia Digital, 3, 1 DOI: https://doi.org/10.33262/cienciadigital.v3i1.348

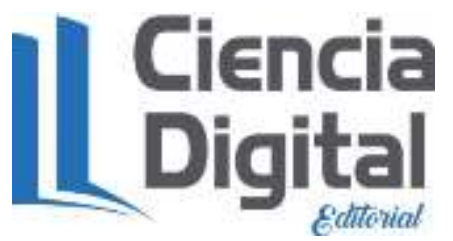




\section{PARA CITAR EL ARTÍCULO INDEXADO.}

Serrano Avalos, K., Villavicencio Narváez, L. del C., Mejía Paredes, M., \& Peñafiel Salazar, D. de los Ángeles. (2019). Discriminación y violencia de género en estudiantes politécnicos en Chimborazo Ecuador. Ciencia Digital, 3(2.6), 104-114. https://doi.org/10.33262/cienciadigital.v3i2.6.521

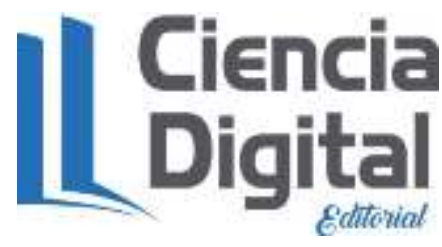

El artículo que se publica es de exclusiva responsabilidad de los autores y no necesariamente reflejan el pensamiento de la Revista Ciencia Digital.

El artículo queda en propiedad de la revista y, por tanto, su publicación parcial y/o total en otro medio tiene que ser autorizado por el director de la Revista Ciencia Digital.
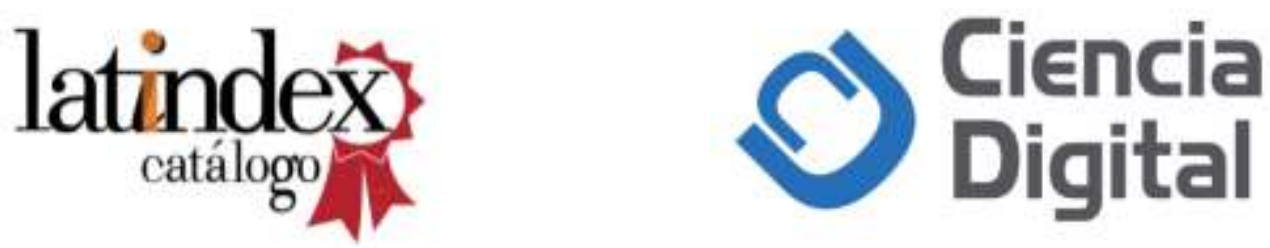